\title{
Relationship between anemia, iron deficiency, and platelet production in dogs
}

\author{
ERDAL MATUR, ELIF ERGUL EKIZ, MERT EREK, EZGI ERGEN, \\ SUAT HAYRI KÜÇÜK**, SONGÜL ERHAN*, MUKADDES ÖZCAN
}

\begin{abstract}
Istanbul University-Cerrahpasa, Faculty of Veterinary Medicine, Department of Physiology, Avcilar, 34320 Istanbul, Turkey
*Istanbul University-Cerrahpasa, Faculty of Veterinary Medicine, Avcilar, 34320 Istanbul, Turkey

**Bagcilar Training and Research Hospital, Medical Biochemistry Department, 34200, Bagcilar, Istanbul, Turkey
\end{abstract}

Matur E., Ergul Ekiz E., Erek M., Ergen E., Küçük S. H., Erhan S., Özcan M. Relationship between anemia, iron deficiency, and platelet production in dogs

Summary

Platelet production increases in anemic people. In particular, iron deficiency causes reactive thrombocytosis in such patients. Although anemia and iron deficiency are frequently seen in dogs, there are no published studies on this subject. The aim of the present study was to investigate the relationship between anemia, iron deficiency, and platelet production in dogs. A total of 81 dogs brought to the university animal hospital with signs of anemia were used. Haemogram parameters, serum iron (Fe), erythropoietin (EPO), and thrombopoietin (TPO) levels, as well as total iron binding capacity (TIBC), were measured. Transferrin saturation (TSAT) ratios were calculated. The animals were categorized as anemic or non-anemic, mildly anemic or moderately/ severely anemic, and having iron deficiency or no iron deficiency according to haemogram parameters and the serum Fe level. A high platelet number was observed in dogs with mild anemia. Dogs with mild anemia also had higher serum EPO levels than dogs with moderate/severe anemia $(P=0.047)$. Iron deficiency was detected in 31 dogs. The platelet number was higher in dogs with iron deficiency $(P=0.004)$. It was also observed that dogs with iron deficiency had higher serum EPO levels $(P=0.027)$ and lower TPO levels $(P=0.025)$ than dogs without iron deficiency. In conclusion, it can be said that mild anemia and iron deficiency cause thrombocytosis in dogs. The increased serum EPO levels, both in dogs with mild anemia and those with iron deficiency, suggest that the increase in platelet production is due to EPO. Nevertheless, there is a need for further research to fully understand the underlying mechanism.

Keywords: dog, anemia, thrombocytosis, iron deficiency, erythropoietin, thrombopoietin

In dogs, as in humans, anemia is one of the most common clinical manifestations. One of the most common causes of anemia in both humans and dogs is iron deficiency (14). Gastrointestinal bleedings, ectoparasites, and inadequate iron intake with food are the main causes of iron deficiency anemia (IDA). IDA is seen more frequently in suckling kids because, although iron demand during the rapid growth period is high, young animals have low iron stores and milk is also low in iron (18). Since these types of anemia develop slowly, clinical signs may not be observed until anemia is significantly advanced. Prominent thrombocytosis in humans with iron deficiency has been reported, but its cause has not been fully understood (8).

Thrombocytosis is classified in two major categories: as essential or reactive (13). Essential thrombocytosis, also called clonal thrombocytosis, is a type of chronic myeloproliferative disorder. In this case, the bone marrow produces too many platelets. Secondary thrombocytosis, or reactive thrombocytosis, occurs due to secondary causes, such as infection, inflammation, tissue damage, hemolysis, severe exercise, malignancy, or hyposplenism (3). The diagnosis and treatment of reactive thrombocytosis due to anemia in humans are quite different from those of clonal thrombocytosis. Therefore, a number of studies have been carried out to elucidate the mechanisms of thrombocytosis due to anemia in humans $(10,20)$.

Marchetti et al. (11) reports that dogs with chronic enteropathy have anemia, and some of these anemic dogs also have thrombocytosis. Reactive thrombocytosis has also been reported in dogs with chronic blood loss (6). However, as far as we know, there have been no studies investigating directly the relationship between anemia and thrombocytosis in dogs. As already mentioned, anemia is one of the most common clinical 
signs in dogs. There are many points worth examining, such as the relationship between the severity of anemia and thrombocyte production, the efficiency of erythropoietin or thrombopoietin mechanisms in the formation of reactive thrombocytosis, or the relationship between reactive thrombocytosis and other blood parameters in anemic dogs. Similarly, the relationship between thrombocytosis and parameters of iron metabolism, such as the serum iron level, total iron binding capacity (TIBC), and the percentage of transferrin saturation (TSAT), has not been investigated in anemic dogs. The elucidation of these details may allow the alterations observed in the number of platelets in anemic dogs to be used as a clinical marker in the diagnosis or prognosis. It can also be determined whether anemiarelated thrombocytosis requires an extra intervention other than anemia treatment. To make all these to be possible, anemia-related thrombocytosis should be studied together with its physiological mechanisms.

The aim of the study was to investigate the relationship between anemia, iron deficiency, and platelet production in dogs. The relationship between platelets and leukocytes will also be discussed.

\section{Material and methods}

Animal material. A total of 81 dogs, which were brought to and blood sampled for routine examinations in the clinics of Istanbul University-Cerrahpasa Veterinary Faculty between 2017 and 2018, were used in the study. This study was approved by the Istanbul University-Cerrahpasa Veterinary Faculty Local Ethics Committee (Approval number 72796624-050.01.01). In order for the blood samples to be used in this study, permission was obtained from the dog owners.

Blood analysis. Two milliliters of anticoagulated (EDTA) and non-anticoagulated blood samples were taken from each dog suspected of having anemia during clinical examination. All samples were processed within three hours. Complete blood count (CBC) was performed in EDTA samples by a cell counter (Abacus Junior Vet, Diatron, Austria) within two hours. Blood samples collected into plain tubes were allowed to cloth, and then they were centrifuged at $3500 \mathrm{rpm}$ for 10 minutes. Serum samples were stored at $-80^{\circ} \mathrm{C}$ for subsequent analysis. Serum iron concentration $(\mathrm{Fe})$ and total iron binding capacity (TIBC) were determined using an auto analyzer and commercial kits. The transferrin saturation ratio (TSAT) was calculated from $\mathrm{Fe}$ and TIBC values as follows: $\mathrm{TSAT}=(\mathrm{Fe} / \mathrm{TIBC}) \times 100$ (4). Serum concentrations of erythropoietin (EPO) and thrombopoietin (TPO) were measured using commercial ELISA kits.

Categorization of animals. The animals were categorized as 1) "anemic" or "non-anemic", 2) "mildly anemic" or "moderately/severely anemic", and 3) having "iron deficiency" or "no iron deficiency" using haemogram parameters and the serum Fe level. The reference values of the cell counter $\left(\mathrm{RBC}=5.5-8.5 \times 10^{6} / \mu \mathrm{L}, \mathrm{HGB}=12-18 \mathrm{~g} / \mathrm{dL}\right.$ and $\mathrm{HCT}=37-55 \%$ ) were used to distinguish between anemic and non-anemic dogs. Dogs with RBC $<5.5 \times 10^{6} / \mu 1$, $\mathrm{HGB}<12 \mathrm{~g} / \mathrm{dl}$, and $\mathrm{HCT}<37 \%$ were considered as anemic.
Anemic dogs were classified into two groups of "dogs with mild anemia", and "dogs with moderate/severe anemia" according to the criteria of Sodikoff (17). According to these criteria, anemia was categorized as mild, moderate, or severe when the HCT values were between $30-40 \%$, $20-30 \%$, or less than $20 \%$, respectively. Dogs with a serum Fe concentration below 81-220 $\mu \mathrm{g} / \mathrm{dl}$ were defined as those with low iron levels (11). Reference values for platelet count $\left(\mathrm{PLT}=200-400 \times 10^{3} / \mu \mathrm{l}\right)$ were used to identify dogs with thrombocytosis.

Statistical analyses. An independent-samples T-test was performed to compare anemic and non-anemic dogs, dogs with mild anemia and dogs with severe anemia, as well as dogs with iron deficiency and dogs without iron deficiency. The Mann-Whitney U test, a non-parametric test, was used to compare parameters that did not show normal distribution according to Levene's test. All statements of significance were based on $\mathrm{P}<0.05$. All statistical analyses were performed using the SPSS Version 21 for Windows (SPSS Inc., Chicago, IL).

\section{Results and discussion}

In the current study, dogs were categorized as anemic or non-anemic considering RBC, HCT, and HGB values. Dogs with RBC, HCT, and HGB values below normal reference ranges were classified as anemic. According to these criteria, $43(53 \%)$ of the dogs included in the study were anemic (21 were mildly anemic, 22 were moderately/severely anemic), whereas $38(47 \%)$ of the dogs were not anemic.

The relationship between the degree of anemia and the platelet count is presented in Fig. 1. It was observed that dogs with mild anemia had a higher platelet count than those with moderate/severe anemia $(\mathrm{P}=0.050)$ (Fig. 1). In addition, the mildly anemic dogs had higher serum EPO levels than those with moderate/ severe anemia $(P=0.047)$ (Fig. 1), However, there was no significant difference in the serum TPO level $(\mathrm{P}=0.112)$, (Fig. 1).

Iron deficiency was detected in $31(38 \%)$ of the dogs included in the study. It was found that 24 of the dogs with iron deficiency were anemic. The dogs

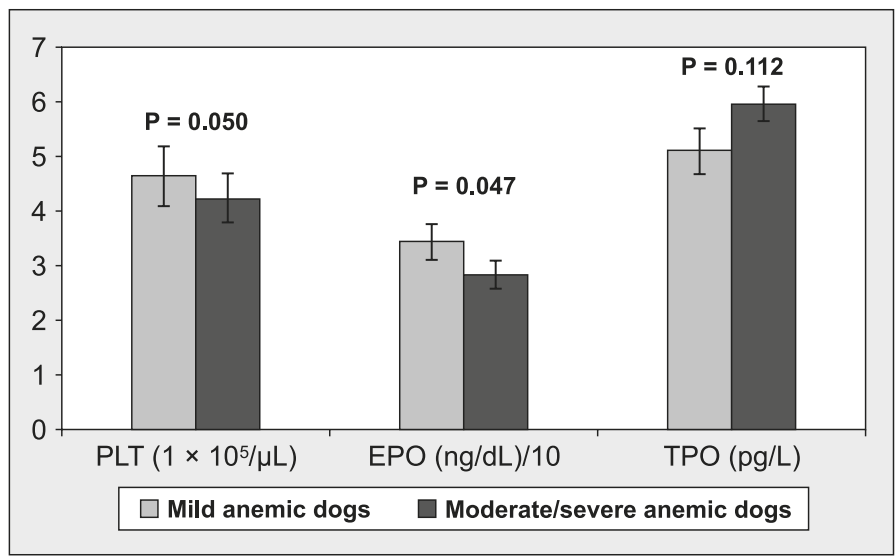

Fig. 1. Relationship between the grade of anemia and the platelet (PLT) number, the serum erythropoietin (EPO) level, and the thrombopoietin (TPO) level 
with iron deficiency had a reactive thrombocytosis, because the number of platelets in iron-deficient dogs was found to be higher than it was in dogs without iron deficiency $(\mathrm{P}=0.004)$ (Fig. 2, 3). In addition, in dogs with iron deficiency, the serum TIBC level was higher $(\mathrm{P}=0.001)$, but the serum TSAT ratio was lower $(\mathrm{P}=0.001)$, (Fig. 2).

It was also observed that dogs with iron deficiency had a higher serum EPO level and a lower TPO level than the dogs without iron deficiency (respectively, $\mathrm{P}=0.027$ and $\mathrm{P}=0.025$ ), (Fig. 3).

Data regarding the relationship between leukocytes and platelets are presented in Table 1. There was no significant difference in terms of the total leukocyte count or the differential leukocyte count between dogs with a normal platelet count and dogs with thrombocytosis $(\mathrm{P}=0.614)$.

In the present study, dogs were categorized with respect to the severity of the anemia. It appears that dogs with mild anemia had reactive thrombocytosis (Fig. 1). It has been suggested that reactive thrombocytosis seen in anemic humans might originate from erythropoietin (2). Indeed, it has been demonstrated both in vitro and in vivo that high-dose EPO administration stimulates platelet production (19). It has also been reported that recombinant EPO, which is used for treatment of anemia in humans, also causes thrombocytosis (9). Biliç and Biliç (2) report that the amino acid sequences of EPO and TPO, especially receptor-binding regions, are very similar, so that EPO can easily cross-react with TPO receptors in the bone marrow. It is therefore suggested that the increased production of platelets in anemic patients is related to EPO, which stimulates platelet production by binding to TPO receptors through cross-reaction (2). As far as we know, there is no published study on dogs regarding this topic. In the current study we observed that dogs with mild anemia tended to have higher serum EPO levels than dogs with moderate/severe anemia. On the other hand, there was no significant difference in the serum TPO level (Fig. 1). This made us think that the increased EPO stimulated platelet production in anemic dogs, as it does in humans.

One of the interesting findings of the current study is that, although thrombocytosis was seen in mildly anemic dogs, there was no thrombocytosis in dogs with moderate or severe anemia (Fig. 1). As already mentioned, we suggest that the thrombocytosis detected in anemic dogs was caused by the binding of EPO to TPO receptors and increased platelet production. While EPO binds to TPO receptors in mildly anemic dogs, the question is why it does not bind to the same receptors in dogs with severe anemia. It is not possible to be certain, but this may be related to the level of EPO receptors expressed in severely anemic dogs. As a matter of fact, an increase in the expression of EPO receptors has been reported in sheep made severely anemic by phlebotomy (5). It has also been reported

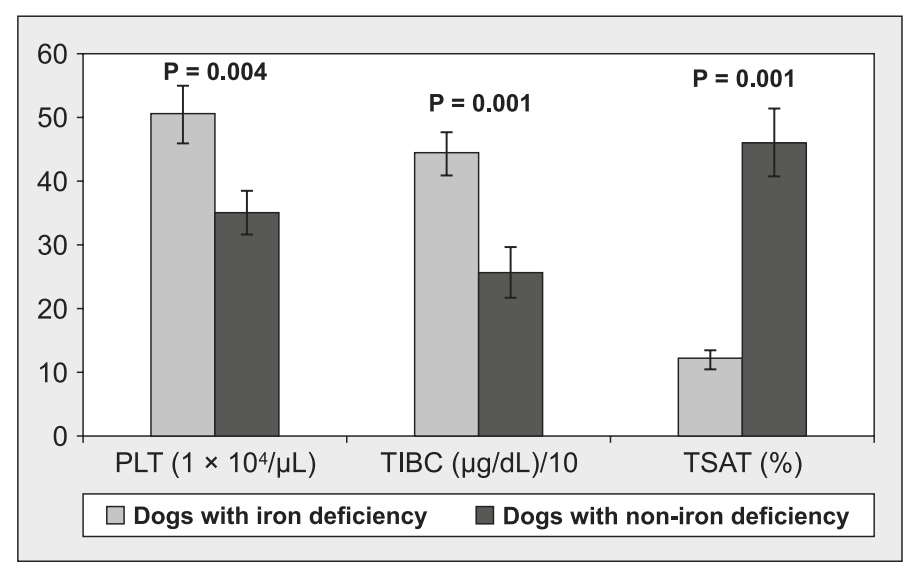

Fig. 2. Platelet numbers, serum total iron binding capacity (TIBC), and transferrin saturation percentage (TSAT) in dogs with iron deficiency and without iron deficiency

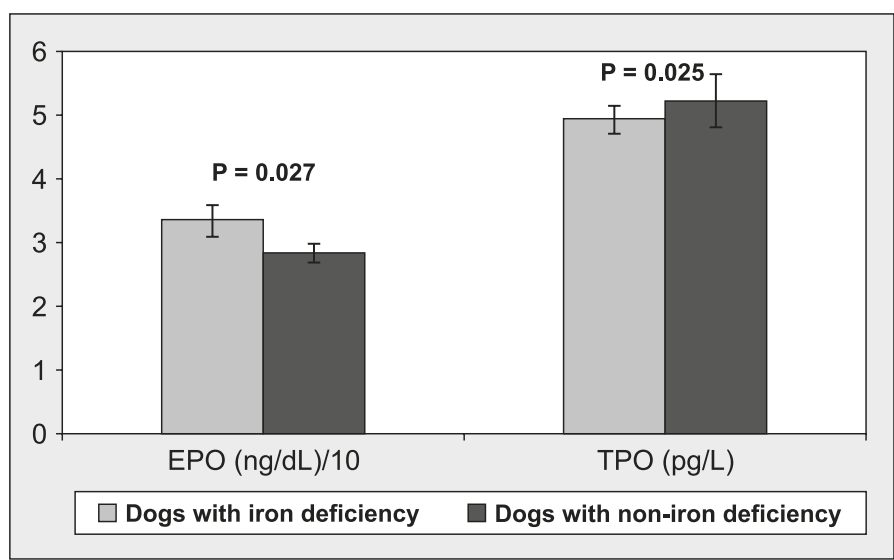

Fig. 3. Serum erythropoietin (EPO) and thrombopoietin (TPO) levels in dogs with and without iron deficiency

Tab. 1. Total and differential leukocyte counts in dogs with normal and increased thrombocyte numbers

\begin{tabular}{|l|c|c|c|}
\hline \multicolumn{1}{|c|}{$\begin{array}{c}\text { Differential } \\
\text { leukocytes }\end{array}$} & $\begin{array}{c}\text { Dogs with a normal } \\
\text { thrombocyte count }\end{array}$ & $\begin{array}{c}\text { Dogs with } \\
\text { thrombocytosis }\end{array}$ & P values \\
\hline WBC $\left(1 \times 10^{3} / \mu \mathrm{L}\right)$ & $21.24 \pm 2.30$ & $23.30 \pm 2.54$ & 0.553 \\
\hline Neutrophil $(\%)$ & $68.89 \pm 2.36$ & $71.82 \pm 3.36$ & 0.456 \\
\hline Lymphocyte (\%) & $24.73 \pm 2.19$ & $22.62 \pm 3.04$ & 0.565 \\
Eosinophil (\%) & $5.10 \pm 1.22$ & $3.72 \pm 0.74$ & 0.459 \\
\hline Monocyte (\%) & $2.72 \pm 0.40$ & $3.62 \pm 0.42$ & 0.142 \\
\hline Basophil (\%) & $1.00 \pm 0.01$ & $2.33 \pm 1.33$ & 0.286 \\
\hline
\end{tabular}

that EPO receptors in the bone marrow are increased in lambs made anemic by phlebotomy, and circulating EPO is eliminated by these receptors (15). As the severity of anemia increases, the number of EPO receptors increases, and these increased EPO receptors eliminate EPO present in circulation. In such a case, there will not be enough EPO bonding to TPO receptors. In the current study, we also observed that serum EPO levels of dogs with severe anemia were lower than those of mildly anemic dogs (Fig. 1).

It has been reported that reactive thrombocytosis in humans is usually seen in anemia due to iron deficiency. It can be said that, in the vast majority of dogs 
included in this study, anemia was caused by iron deficiency, since 31 of them (38\%) seemed to have iron deficiency. Serum TIBC and TSAT levels also support this idea (Fig. 2). In dogs with iron deficiency, a high serum TIBC level combined with a low TSAT ratio was a typical finding.

In the present study, the platelet number in dogs with iron deficiency was significantly higher than it was in dogs with a normal serum iron level (Fig. 2). This made us think that there is an important relationship between iron metabolism and platelet production in dogs, as well. In studies conducted in humans, the relationship between iron deficiency and platelets has been demonstrated. However, its mechanism has not yet been fully understood $(7,16)$. The fact that the EPO level in dogs with iron deficiency in our study was higher than it was in dogs with a normal serum iron level ( $\mathrm{P}=0.027$, Fig. 3$)$ strengthens the idea that the increase in the number of platelets may be related to EPO. Likewise, in people treated with iron, both the decrease in EPO and the return of the platelet count to normal values (1) support the idea that the mechanism works through EPO.

In this study, it was observed that serum TPO levels were lower in dogs with iron deficiency than in those without iron deficiency, despite an increase in the platelet count $(\mathrm{P}=0.025$, Fig. 3$)$. This seems to be controversial, since the most important regulator of platelet production in megakaryocytes is TPO. However, TPO binds to c-mpl receptors present on the surface of both megakaryocytes and platelets (12). In this case, when the number of platelets in peripheral blood is increased, the value measured in serum may be low, since some of the produced TPO will bind to thrombocytes.

The relationship between leukocyte production and thrombocytosis was also examined in the present study (Tab. 1), but no link was found between leukocyte production and the thrombocytosis mechanism.

In this study, reactive thrombocytosis was detected in dog with mild anemia, but not in dogs with severe or moderate anemia. It was observed that iron deficiency caused thrombocytosis in dogs, as it does in humans. The increased serum EPO levels, both in dogs with mild anemia and in those with iron deficiency, suggest that the increase in platelets is due to EPO. It is impossible, however, to explain the mechanism exactly on the basis of these data. Since platelet production is a complex mechanism, there is a need for further research in which EPO and TPO receptors, cytokines, and growth factors are also taken into account.

\section{References}

1. Akan H., Güven N., Aydogdu I., Arat M., Beksaç M., Dalva K.: Thrombopoietic cytokines in patients with iron deficiency anemia with or without thrombocytosis. Acta Haematol. 2000, 103, 152-156.

2. Bilic E., Bilic E.: Amino acid sequence homology of thrombopoietin and erythropoietin may explain thrombocytosis in children with iron deficiency anemia. J. Pediatr. Hematol. Oncol. 2003, 25, 675-676.

3. Dan K.: Thrombocytosis in irondeficiency anemia. Internal Med. 2005, 44, 1025-1026.
4. Eleftheriadis T., Liakopoulos V., Antoniadi G., Stefanidis I.: Which is the best way for estimating transferrin saturation? Ren. Fail. 2010, 32, 1022-1023.

5.Freise K. J., Widness J. A., Segar J. L., Schmidt R. L., Veng-Pedersen P.: Increased erythropoietin elimination in fetal sheep following chronic phlebotomy. Pharmacol. Res. 2007, 24, 1653-1659.

6. Giger $U$.: Regenerative anemias caused by blood loss or hemolysis, [in:] Ettinger S. J., Feldman E. C. (ed): Textbook of Veterinary Internal Medicine. Elsevier Saunders, St. Louis 2005, p. 1886.

7. Holbro A., Volken T., Buser A., Sigle J. P, Halter J. P., Passweg J. R., Tichelli A., Infanti L.: Iron deficiency and thrombocytosis. Vox Sang. 2017, 112, 87-92.

8. Kadikoylu G., Yavasoglu I., Zahit Bolaman Z., Senturk T.: Platelet parameters in women with iron deficiency anemia. J. Natl. Med. Assoc. 2006, 98, 398-402.

9. Kaupke C. J., Butler G. C., Vaziri N. D.: Effect of recombinant human erythropoietin on platelet production in dialysis patients. J. Am. Soc. Nephrol. 1993, 3, 1672-1679.

10. Kim S., Cho S. Y.: Investigation of iron metabolism for regulating megakaryopoiesis and platelet count according to the mechanisms of anemia. Clin. Lab. 2018, 64, 329-332.

11. Marchetti V., Lubas G., Lombardo A., Corazza M., Guidi G., Cardini G.: Evaluation of erythrocytes, platelets, and serum iron profile in dogs with chronic enteropathy. Vet. Med. Int. 2010, 28, 1-5.

12. Mitchell O., Feldman D., Diakow M., Sigal S.: The pathophysiology of thrombocytopenia in chronic liver disease. Hepat. Med.: Evid. Res. 2016, 8, 39-50.

13. Mizukoshi T., Fujino Y., Yasukawa K., Matumoto H., Matsumura S., Nagasaki T. Ohno K., Tsujimoto H., Shimoda T.: Essential thrombocythemia in a dog. J. Vet. Med. Sci. 2006, 68, 1203-1206.

14. Naigamwalla D. Z., Webb J. A., Giger U.: Iron deficiency anemia. Can. Vet. J 2012, 53, 250-256.

15. Nalbant D., Saleh M., Goldman F. D., Widness J. A., Veng-Pedersen P.: Evidence of receptor-mediated elimination of erythropoietin by analysis of erythropoietin receptor mRNA expression in bone marrow and erythropoietin clearance during anemia. J. Pharmacol. Exp. Ther. 2010, 333, 528-532.

16. Schloesser L. L., Kipp M. A., Wenzel F. J.: Thrombocytosis in iron-deficiency anemia. J. Lab. Clin. Med. 1965, 66, 107-114.

17. Sodikoff C. H.: Laboratory profiles of small animal diseases. Mosby Inc, St Louis 2001.

18. Stockholm S. L., Scott M. A.: Fundamentals of Veterinary Clinical Pathology. Blackwell Publishing, Ames Iowa 2002, p. 107.

19. Vaziri N. D., Zhou X. J.: Potential mechanisms of adverse outcomes in trials of anemia correction with erythropoietin in chronic kidney disease. Nephrol. Dial. Transpl. 2009, 24, 1082-1088.

20. Voigt W., Jordan K., Sippel C., Amoury M., Schmoll H. J., Wolf H. H.: Severe thrombocytosis and anemia associated with celiac disease in a young female patient: a case report. J. Med. Case Reports. 2008, 2, 96.

Corresponding author: Prof. Dr. Erdal Matur, Istanbul UniyersityCerrahpasa, Faculty of Veterinary Medicine, Department of Physiology, 34320, Istanbul, Turkey; e-mail: mature@istanbul.edu.tr 